\title{
Neue Unübersichtlichkeit in den Unterrichtswissenschaften
}

Ich möchte in dem folgenden Beitrag den Zusammenhang zwischen gesellschaftlichen Entwicklungen, erziehungswissenschaftlicher (pädagogischer) Theoriebildung, allgemeiner Didaktik und Fachdidaktik der letzten Jahrzehnte skizzieren. Dabei beginne ich mit der Situation in den fünfziger Jahren und gehe chronologisch vor. Aus dieser sowohl historisch als auch differentiell verfahrenden Betrachtungsweise möchte ich meine These ableiten und begründen: Der gegenwärtige Zustand der Unterrichtswissenschaften (1) ist als neue Unübersichtlichkeit zu charakterisieren.

\section{Die geisteswissenschaftliche Pädagogik am Ausgang ihrer Epoche}

Um sich deutlich zu machen, welches pädagogische Selbstverständnis im deutschsprachigen Raum der Nachkriegszeit, also bis einschließlich der 50er Jahre geherrscht hat, muß man sich die gesellschaftliche Realität der Schule der vorfaschistischen Zeit vor Augen halten. Unmittelbar nach dem 2. Weltkrieg wurde nämlich in pädagogischer und didaktischer Theorie und Praxis auf das zurückgegriffen, was bis dahin, also in den ersten Jahrzehnten des Jahrhunderts, entwickelt wurde.

Die Schule des 19. und des beginnenden 20. Jahrhunderts war davon geprägt, daß es in ihr nicht eigentlich Sitzenbleiber gegeben hat. Wer ins Gymnasium eintrat, der blieb mit hoher Wahrscheinlichkeit bis zum Abschluß dort. Gesellschaftlich betrachtet, bedeutet dies: die Differenzierung der Bildungsbzw. der Schulabschlüsse ergab sich sozialständisch. Wer auf welche Schule ging, wurde nach der gesellschaftlichen Stellung des Vaters geregelt. Bis lange ins 20. Jahrhundert funktionierte dieser gesellschaftliche Differenzierungsmodus. Er löste sich nur langsam auf und blieb bis in die fünfziger Jahre relativ stabil.

Dort, wo er sich etwa im ausgehenden 19. Jahrhundert aufzulösen drohte, gab es rigorose staatliche Eingriffe, wie das folgende Zitat Gosslers, in den 90er Jahren des vorigen Jahrhunderts Kultusminister in Preußen, zeigt: Gossler lastet die damals drohende Bildungskatastrophe «dem falschverstandenen Bildungstrieb der Eltern, den kleinen Grundbesitzern, Subaltern- und Unterbeamten, kleinen Ge- werbetreibenden und Handwerkern an, die nicht den Mut oder die Einsicht haben, ihre Kinder dem Stande wieder zuzuführen, aus dem sie hervorgegangen sind ... Infolge der Erschütterung auf wirtschaftlichem Gebiet werden eine Menge Elemente den Gymnasialanstalten zugeführt..., die früher sicher bei Handel, Gewerbe und Industrie ihr Unterkommen gesucht und gefunden hätten, jetzt aber einer Gelehrtenbildung und namentlich dem Beamtentum zustreben.» (2)

Der preußische Staat - er soll hier nur exemplarisch für dieses Problem stehen - betrieb eine Politik, in der das staatserhaltende Kleinbürgertum gestärkt wurde, indem man den Ausbau des mittleren und des Fachschulwesens forcierte und so gleichzeitig die mittleren sozialen Schichten von der Eliteanstalt des Gymnasiums fernhielt. Damit ist exemplarisch der Mechanismus der Entwicklung unseres europäischen Schulsystems benannt: es differenzierte sich sozialständisch. Damit war die Schule weitgehend von einer gesellschaftlichen Selektionsfunktion entbunden.

Die dieser Realität korrespondierende pädagogische Theorie ist die geisteswissenschaftliche Pädagogik.

Die Geisteswissenschaften entstehen auf dem Höhepunkt der industriellen Umgestaltung Kontinentaleuropas, also in einem Augenblick, in dem die Naturwissenschaften durch ihre technische Anwendung unser Leben entscheidend verändern. Die Geisteswissenschaften konstituieren einen neben den Naturwissenschaften existierenden eigenen Erkenntnis- und Wissenschaftsbereich, der, so drückt es Dilthey programmatisch aus, im Gegensatz zu den Naturwissenschaften, die ihre Gegenstände erkennen, ihre Objektivationen, wie das Dilthey nennt, nicht erkennen, sondern verstehen. Die Geisteswissenschaften sind also die eigentlichen Kulturwissenschaften.

Diese Entwicklung eines eigenständigen Forschungsbereichs mit eigenen wissenschaftlichen Methoden, deren zentralste die Hermeneutik, also

H.J. Forneck, Dr., Direktion der Sekundar- und Fachlehrerausbildung an der Universität Zürich, Voltastr. 59, 8044 Zürich. 
die Kunst der Sinnauslegung ist, wird nun von Hermann Nohl zu Beginn unseres Jahrhunderts zu einer eigenen wissenschaftlichen Pädagogik ausgearbeitet.

Die geisteswissenschaftliche Pädagogik geht von der Erziehungswirklichkeit aus. Das wird aber nicht so verstanden, wie wir heute Wirklichkeit verstehen würden. Sondern die pädagogische Wirklichkeit wird als ein sinnvolles Ganzes, als ein selbständiges Kultursystem, aufgefaßt, hinter dem «die neue soziale, sittliche und geistige Not unseres Volkes, die durch die Entwicklung der Industrie, der Großstädte, der Arbeits- und Wohnverhältnisse, aber auch durch die naturwissenschaftliche Aufklärung und die Entwicklung der Spezialwissenschaften über die Seelen hereingebrochen ist. In diesem Prozeß ging der Selbstwert des Subjekts verloren; der Mensch, der nur noch nach seiner Leistungskraft gewürdigt wurde, wurde zur Masse, . . .» (3)

Auf diese geistige Krise der Zeit gab es nach Nohl eine Bewegung, die die Landschulbewegung, die pädagogische Reformbewegung, die Volkshochschulbewegung, die Frauenbewegung, die (vornehmlich katholische) soziale Bewegung umfaßt und die Nohl die deutsche Bewegung nennt.

Nohl arbeitet nun die Implikationen dieser Bewegung, besser die Implikationen ihrer Praxis, verstehend heraus. In diesem methodischen Vorgehen ist bereits das grundlegende Theorie-Praxis-Verständnis der geisteswissenschaftlichen Pädagogik enthalten. Praxis geht Theorie danach immer voraus. Theorie folgt dieser Praxis nach, erforscht diese, indem sie sie versteht, ihre Gedanken explizit macht. Der Praktiker, der sich dann mit Theorie beschäftigt, versteht durch die Beschäftigung mit der geisteswissenschaftlichen Pädagogik seine eigene Praxis besser.

Das heißt aber nun auch, daß Theorie nie über Praxis hinausgehen kann. Praxis ist immer weiter als Theorie, ist immer reicher, fortschrittlicher. Das Schöpferischste, Wesentlichste liegt in der Praxis verborgen. Theorie kann nur das, was schon vorhanden ist, zum Vorschein bringen, Theorie kann selbst nie Praxis begründen.

In der Analyse der pädagogischen Bewegung arbeitet Nohl dann das Wesentliche des Pädagogischen heraus: Erziehung ist immer ein einzigartiges Verhältnis zwischen zwei Menschen. «Die Grundlage der Erziehung ist also das leidenschaftliche Verhältnis eines reifen Menschen zu einem werdenden Menschen, und zwar um seiner selbst willen, daß er zu seinem Leben und seiner Form komme.» (4) Dieses Verhältnis nennt die geisteswissenschaftliche Pädagogik den pädagogischen Bezug, welcher für Nohl auf einer instinktiven Grundlage basiert. Der Erzieher versucht mit Hingabe eine Beziehung aufzubauen, die von Liebe getragen ist. Dieses Ethos des pädagogischen Verhältnisses hat ein Ziel: «aus diesem Kinde machen, was aus ihm zu machen ist, das höhere Leben in ihm entfachen und zu zusammenhängender Leistung führen, nicht um der Leistung willen, sondern weil in ihr sich das Leben des Menschen vollendet.» (5)

Der Lehrer hat also in seiner Berufstätigkeit die Perspektive des Kindes einzunehmen - und dies in doppelter Weise: er muß dieses Individuum in seiner Einmaligkeit verstehen, und er muß die $\mathrm{Zu}$ kunft des Kindes stellvertretend für das Kind denken, da dieses die Zukunft noch nicht denken kann. Er muß letzteres tun, um die Schule von Anforderungen und Ansprüchen fernzuhalten - z. B. von Staat, Kirche und Wirtschaft -, die die Zukunft des Kindes zu sehr festlegen. Deshalb formuliert Nohl: «Unabhängig von den Ansprüchen, die der Beruf oder sonst irgendwelche objektiven Mächte des Lebens an uns stellen, soll hier das Menschliche sich erfüllen.»(6) Daraus entsteht die Forderung nach der Autonomie der Pädagogik. (7)

Das Ziel der geisteswissenschaftlichen Pädagogik ist die autonome Persönlichkeit. Ihr Mittel ist die Bildung. «Bildung ist die subjektive Seinsweise der Kultur, die innere Form und geistige Haltung der Seele, die alles, was draußen an sie herankommt, mit eigenen Kräften zu einheitlichem Leben in sich aufzunehmen und jede Äußerung und Handlung aus diesem einheitlichen Leben zu gestalten vermag.» (8)

In den Lernzielen für den Geographieunterricht schlägt sich eine solche Auffassung nieder. So bemerkt etwa Schmidt 1972 in einer Analyse der Lehrpläne: «In den Lehrplänen der jüngeren Vergangenheit (9) finden sich weiterhin nicht selten emotionale Ziele der Erdkunde. So sprach Baden-Württemberg... vom Staunen vor der Schönheit der Erde und von der Ehrfurcht vor dem Bau des Weltalls. Schleswig-Holstein ... formulierte, mit der Heimat im Herzen die Welt umfassen und mit der Welt vor Augen die Heimat liebend und bauend durchdringen. Rheinland-Pfalz . . . stellte die Führung des jungen Menschen von der Heimatliebe zu besonnener Vaterlandsliebe heraus. In NordrheinWestfalen... sollte die Himmelkunde zur Ehrfurcht vor dem Schöpfer erziehen.» (10) Die Gewinnung eines erdkundlichen Weltbildes, eines der Hauptanliegen des Geographieunterrichts, wird hier verknüpft mit kulturellen Interpretationen der Bedeutung von Natur, Erde und Weltall.

Mit einem solchen, von der gesellschaftlichen Funktionalität abgekoppelten Bildungsbegriff und gesellschaftlich nicht funktionalisierten Unterrichtsinhalten bin ich argumentativ wieder am Anfang, nämlich der gesellschaftlichen Stellung und Funktion der Schule angelangt. Solange nämlich die Schule, zumindest ihre allgemeinbildenden Zweige, keine unmittelbaren gesellschaftlichen Funktionen zu erfüllen hatten, konnten eine solche Pädagogik und die korrespondierende didaktische Theorie und unterrichtliche Praxis bestehen. 
Die gesellschaftliche Funktion der Schule ändert sich aber nun grundlegend.

\section{Die realistische Wende in den Unterrichtswissenschaften}

Ende der sechziger Jahre findet in der deutschsprachigen erziehungswissenschaftlichen Diskussion eine Auseinandersetzung um die Professionalisierung des Lehrerberufs statt, in der davon ausgegangen wird, «daß die Professionalisierung des Lehrerberufs als einer der konstitutiven Massenberufe der modernen „entwickelten” Gesellschaft im Gegensatz zur Professionalisierung anderer vergleichbarer Berufe wie z. B. des Arztes oder auch des Verwaltungsfachmannes oder des Ingenieurs nicht eigentlich gelungen ist ...» (11) Die Professionalisierungsdiskussion ist das in der bildungspolitischen Öffentlichkeit wohl deutlichste Zeichen für eine Anbindung der Schule an gesellschaftliche Bedürfnisse. Im Bereich der Unterrichtswissenschaften wird dieser Proze $ß$ als die «realistische Wende» bezeichnet.

Um nun diese Entwicklung der Unterrichtswissenschaften, die sich ungeheuer schnell durchsetzt, zu verstehen, müssen wir uns des Datums, das hier angesprochen ist, genau vergewissern:

Am 4.10.1957 gelingt es der Sowjetunion, den ersten Satelliten in eine Umlaufbahn zu schießen. Die westliche Welt erleidet den sog. Sputnikschock. Inhaltlich bedeutete dieses Ereignis, daß der breiten westlichen Öffentlichkeit bewußt wurde, daß die sozialistischen Staaten einen wissenschaftlichen und technologischen Vorsprung hatten, ein Faktum, das in Expertenkreisen auch für den schulischen, bzw. pädagogischen Bereich bekannt war. In den fünfziger Jahren hatten z. B. die Berliner Didaktiker Heiman, Otto und Schulz entdeckt, daß die an der sowjetischen didaktischen Theorie und Praxis orientierte Schule der DDR sehr viel weiter als die westeuropäischen Schulen in der Entwicklung der didaktischen Theorie und Praxis fortgeschritten waren.

Der Westen reagierte auf dieses Faktum mit einem quantitativen und qualitativen Ausbau des Bildungswesens ohnegleichen. Historisch ist diese Entwicklung einmalig. Begabungsreserven sollten mobilisiert werden, dazu sollte ein neues Bildungssystem dienen, das sehr viel durchlässiger sein sollte als das drei- oder viergliedrige alte System.

Qualitativ sollte der Unterricht auf eine wissenschaftliche Grundlage gestellt werden. Es entstanden nun Lehrstühle für Erziehungswissenschaft, die sich nicht mehr geisteswissenschaftlich, also die Unterrichtspraxis verstehend, begriffen, sondern die mit Hilfe großangelegter empirischer Untersuchungen und mit statistischen Instrumentarien die Effi- zienz des Unterrichts untersuchten und zu verbessern trachteten.

In dieser Zeit entsteht die europäische Sozialisationsforschung, die die familiären und milieubedingten Defizite der intellektuellen Leistungsfähigkeit freilegt. In den Nachbardisziplinen entsteht die Sprachbarrierentheorie usw.

Innerhalb weniger Jahre etabliert sich so eine reichhaltige, interessante Forschungspraxis und eine Fülle von Schulversuchen.

Der Lehrerberuf gerät allerdings mit dieser Entwicklung zunehmend unter Druck: Zwar wird der Beruf finanziell und von seiner gesellschaftlichen Bedeutung aufgewertet (12) - aber zugleich erwartet man vom Lehrer, daß er der eigentliche Motor dieser technologischen und wissenschaftlichen Kraftanstrengung ist, in der die westlichen Gesellschaften den Osten überholen wollen - und dies auch getan haben. Das Bildungswesen soll nun in einen funktionellen Zusammenhang mit der gesellschaftlichen bzw. mit der ökonomischen Entwicklung gebracht werden. (13)

Der Lehrer soll nun all das, was an didaktischen, methodischen, erziehungswissenschaftlichen, fachwissenschaftlichen, sprachwissenschaftlichen, sozialisationstheoretischen Kenntnissen vorliegt, in seinem Unterricht berücksichtigen.

Es entstehen ganz neue Strukturen in der Schule: z. B. Förder- und Stützkurse, Leistungsniveaus, Teamteaching usf.

Das alte pädagogische Thema der Förderung des Schülers wird zum Zweck der Mobilisierung der gesellschaftlichen Begabungsreserven zum zentralen Thema in unserem Schulsystem.

Mit einem Satz: die Schule erhält eine eminent zentrale gesellschaftliche Funktion; sie wird als ökonomischer Faktor entdeckt. Es ist klar, daß die «Erweckung eines einheitlichen geistigen Lebens durch ein anderes einheitliches geistiges Leben", oder wie immer die geisteswissenschaftliche Pädagogik das leidenschaftliche Verhältnis eines Erziehers zu seinem Zögling beschrieb, ab den sechziger Jahren Rationalisierungskriterien unterworfen wird.

Die Forderung nach der Professionalisierung des Lehrerberufs verweist auf grundlegende Veränderungen in der gesellschaftlichen Bedeutung der Schule und im beruflichen Selbstverständnis des Lehrers einerseits und zeigt andererseits paradigmatische Veränderungen innerhalb der Pädagogik und Didaktik an, die sich bereits zu Beginn der sechziger Jahre abzeichnen.

Professionalisierungsprozesse zeichnen sich durch die «inhaltliche Durchdringung von gesellschaftlich entscheidenden Berufsrollen durch das Erfordernis systematischer wissenschaftlicher Kenntnisse» aus, damit «immer mehr Positionsträger . . . in der Lage sind, vor neue Situationen gestellt, diese zu durchleuchten sowie selbständig und rasch durch Verfügung über wissenschaftliche Hilfsmittel die richti- 
gen Entscheidungen zu treffen.» (14) War bis dahin ein geisteswissenschaftlich orientierter Bezug auf die pädagogische Praxis maßgebend, in dem die Dignität der Praxis der Theorie vorgelagert war und letztere immer nur das reflexiv erhellen sollte, was in der Praxis selbst schon enthalten war, so ist mit der Professionalisierungsforderung erziehungswissenschaftlicher Theorie die Aufgabe zugewiesen, Praxis zu effektivieren und zu optimieren. Im Fachgebiet vollzieht sich der Wechsel zu realwissenschaftlichen Disziplinen, erkennbar an der Etablierung von Unterrichtsdisziplinen an Universitäten.

Diese veränderte Funktionsbestimmung von pädagogischer Theorie und Praxis und die damit einhergehende Krise des Lehrerberufs und der Schule ist angemessen nur auf dem Hintergrund einer veränderten ökonomischen Situation zu verstehen. Die Krise des Lehrerberufs und der Schule fällt mit dem in den 60er Jahren wichtiger werdenden Tatbestand zusammen, daß der wirtschaftliche Wohlstand einer Nation entscheidend von ihrer geistigen Produktivkraft abhängig ist: «Verschiedene Gründe sprachen so für eine verstärkte Einführung und Anwendung des technischen Wandels... Mit gewissem Recht kann - auf diese Zeit (1955-1960) bezogen - von einer Besinnung der Unternehmer auf den technischen Fortschritt gesprochen werden.»(15) Geistige Produktivkraft ist wesentlich in der Schule beeinflußbar. Damit werden Unterricht und Lehrertätigkeit zu gesellschaftlich entscheidenden Faktoren der Hervorbringung der geistigen Produktivkraft einer Gesellschaft.

Auf diesen gesellschaftlichen Hintergrund ist die ab den 60er Jahren geführte fachwissenschaftliche Diskussion über den «,,Bildungsnotstand”, die „Bildungskatastrophe" usw.» zu verstehen. «Gegenstand der Kritik (sind) das veraltete Schulsystem..., der Qualifikationsrückstand gegenüber anderen Ländern, die „,technologische Lücke”, die fehlenden Mittel für Bildung und Wissenschaft, der Mangel an Lehrern und Naturwissenschaftlern.» (16)

Entsprechend dieser Entwicklung wird in der Lehrerausbildung die Qualifikationsfrage nach der "realistischen Wende" in der Erziehungswissenschaft auf dem Hintergrund eines empirischen Wissenschaftsverständnisses verhandelt. Gefordert wird eine Hinwendung zum realen Erzieher, der nicht idealistisch überhöht werden dürfe. Der idealistische Impetus des Lehrerberufs, wie ihn etwa die geisteswissenschaftliche Pädagogik vertrete, habe die Problematik des Berufes vergrößert. (17) Gesellschaftlicher Optimierungsanspruch fällt hier mit einem zumeist szientifisch verkürzten Wissenschaftsverständnis zusammen. Das Verhältnis von Erziehungswissenschaft und unterrichtlicher Praxis wird in Anlehnung an das Verhältnis von Naturwissenschaft und Technik konzipiert. (18) Damit ist ein neues Verständnis des Lehrerberufs konstituiert, worin der Lehrer als Experte für Unterrichtsprozesse erscheint.

Die didaktische Entwicklung vollzieht die hier angesprochene erziehungswissenschaftliche Entwicklung mit. Hatte die bildungstheoretische Didaktik, in der wissenschaftstheoretischen Tradition der geisteswissenschaftlichen Pädagogik stehend, noch ihre didaktischen Entscheidungen um den Bildungsbegriff zentriert und mit Hilfe der didaktischen Analyse «auch den Kern der Unterrichtsvorbereitung und damit das Fundament der alltäglichen Schularbeit»(19) in eben jener bildungstheoretischen und zugleich normativen Fundierung des Unterrichts gesehen, so wird der Bildungsbegriff mit der realistischen Wende durch den Lernbegriff abgelöst: «Der pädagogische Wert des Unterrichts liegt ausschließlich in seinem Einfluß auf Lernprozesse, in den Anpassungsleistungen, die er bei den Lernenden bewirkt.» (20) Am Bildungsbegriff werden die normativen Konnotationen abgelehnt, die durch den wertfreien Lernbegriff vermieden werden sollen. Allerdings wird dieser «Zuwachs an Wertfreiheit», der mit dem metatheoretischen didaktischen Modell gegeben ist, welches die lerntheoretische Didaktik darstellt, durch einen Verlust an integrativem Potential erkauft. Die allgemeine Didaktik stellt nun keine inhaltlich aufgeladenen allgemeinen Kategorien und Prinzipien mehr zur Verfügung, an denen sich die Fachdidaktik orientieren und in deren Horizont sie die Inhalte des Unterrichts bestimmen könnte. Hier liegt die allgemeindidaktische Ursache der zunehmenden Orientierung des Unterrichts an den Fachwissenschaften, denn inhaltlich bedeutsame Kriterien auch zur inhaltlichen Unterrichtsgestaltung sind nun nur noch bedingt aus den metatheoretischen Überlegungen der lerntheoretisch ausgerichteten Allgemeinen Didaktik zu erhalten. Eine solche Ausrichtung der Unterrichtsinhalte an den fachwissenschaftlichen Disziplinen ist allerdings gewollt. Nicht mehr steht die Selbstverständigung des Individuums in einer orientierungsbedürftigen Welt im Zentrum des schulischen Auftrags. Vielmehr geht es um eine gesellschaftliche Funktionalisierung des Schulwesens. Damit aber ändert sich auch die Aufgabe der Didaktik. Ihr Schwergewicht verlagert sich auf die Optimierung des Unterrichts durch eine Verbesserung der Methodik. Zwar taucht in der Strukturanalyse der lerntheoretischen Didaktik die Intentionalität als eines der sechs Strukturelemente auf, in dem die pädagogischen Absichten aufgenommen sind; der eigentliche Charakter der lerntheoretischen Didaktik wird auf der zweiten didaktischen Reflexionsstufe, auf der über das in den sechs Strukturelementen Erfaßte nachgedacht wird, deutlich: «Auf dieser Stufe (der zweiten didaktischen Reflexionsstufe HJF) wird das Wissen aktualisiert werden müssen, das die pädagogischen Grundwissenschaften und 
das Wahlfachstudium bereitstellen. Z. B. werden zur Normenkritik Denkmodelle der imperativen Logik, der Semantik, der Wissenssoziologie und der Psychoanalyse herangezogen. Die anthropologischen Disziplinen helfen, den Stand der faktischen Orientierung in bezug auf die angenommenen Voraussetzungen bei Mensch und Gesellschaft zu überprüfen. Die Sachgemäßheit der Unterrichtsstrukturierung wird mit Hilfe der sogenannten Wahlfächer (21) geprüft, die Lerneffektivität unter Verwendung von Ergebnissen der Lernforschung . . .» (22) In solchen Passagen wird die gesellschaftliche Funktionalisierung der Schule auf didaktischer Ebene reproduziert.

Folglich kann Schmidt auch für die Veränderung der Lernziele für den Geographieunterricht feststellen: «Die moderne Erdkunde in den Lehrplänen der Gegenwart zeigt also eine deutliche Tendenz zur Versachlichung, zur Lösung vom Emotionalen.» (23) Daneben ist eine pragmatische Tendenz in den Lehrplänen nicht zu übersehen, die auf eine «Bewältigung bestimmter Lebenssituationen in der Zukunft des jungen Menschen» zielt. (24)

Allerdings ist mit solchen ausschließlich «sachlich», was ja eine Abkehr von normativen Zugriffen auf das Schulsystem meint, das komplexe Verhältnis zwischen Bildungssystem und geistiger Produktivkraftsteigerung einerseits und erzieherisch relevanter Theorie und unterrichtlicher Praxis andererseits nicht faßbar.

Bevor sich allerdings dieses Programm der realistischen Wende in der Schulpraxis durchschlagend hat durchsetzen können, bricht eine neue Entwicklung in den westlichen Industrienationen auf, die soziologisch ein neues Phänomen hervorbringt: die Verbindung von sozialistischem Gedankengut und Intelligenz. Gemeint ist hier die Studentenbewegung, die für erziehungswissenschaftliche und didaktische Theoriebildung einerseits und unterrichtlicher Praxis andererseits nicht unbedeutend geblieben ist.

\section{Kritisch-emanzipative Wende und resignative Atomisierung}

Während nun in den Unterrichtswissenschaften relativ spät und nicht durchgängig die lange verdrängte Auseinandersetzung mit dem empirischanalytischen Wissenschaftsverständnis der realistischen Wende und den sich daraus ergebenden unterrichtlichen Konsequenzen aufgenommen wird, sind in der sozialwissenschaftlichen Diskussion Positivismusstreit und Technokratiediskussion entbrannt, die «kritisch-emanzipatorische Wende» zeichnet sich $a b$.

Das Wertfreiheitspostulat wird nun unter gesellschaftskritischer Perspektive rückgängig gemacht, und die Unterrichtswissenschaften werden um den Begriff der Emanzipation zentriert. Dabei gerät dieser Versuch allerdings in einen Zirkel, denn was sich aus dem Erziehungsziel «Emanzipation» im «Kontext pädagogischen Handelns» als Zwischenoder Teilziele ergibt, kann keine Theorie mit Bestimmtheit sagen, es sei denn, das, was für den Begriff «Emanzipation» als unverzichtbar behauptet wird, nämlich die Chance für Individuen und Gruppen, ihr Handeln selbst zu bestimmen, würde aufgegeben (...). (25) Dieses Dilemma einer emanzipatorischen Pädagogik kann immer nur nachträglich, also «in gelingenden Prozessen der Aufklärung und d. h.: im praktischen Diskurs der Betroffenen eingelöst werden». (26) Nun könnte man meinen, durch diese logische Ausweglosigkeit einer emanzipatorischen Erziehung würde die integrative Wirkung, auf die eine emanzipatorische Pädagogik zielte, bereits im Ansatzpunkt wieder rückgängig gemacht. Für die didaktische Theoriebildung ist dies allerdings nur bedingt gültig. Zwar zeigt es sich, daß die Bestimmung von emanzipatorischen Bildungsinhalten je nach dem gesellschaftskritischen Standort der Analyse differiert und somit so viele Schattierungen von emanzipatorischen Bildungsinhalten wie gesellschaftskritische Standpunkte existieren. Im Bereich der Unterrichts- und Sozialformen ergeben sich allerdings mit der «emanzipativen Wende» sehr schnell integrative Wirkungen. Dem Frontalunterricht wird nun generell eine emanzipationshemmende Wirkung zugesprochen, während man den Projektunterricht als eine fortschrittliche Methode ansieht. Das Attribut fortschrittlich wird auf der Grundlage einer nun emanzipatorisch interpretierten Lerneffektivität verstanden: Der Projektunterricht ermögliche das Durchschauen einer immer komplexen - weil gesellschaftlich vermittelten - Wirklichkeit. Ein solcher sich auf den verschiedenartigsten Ebenen und in den unterschiedlichsten fachwissenschaftlichen Disziplinen abspielender Projektunterricht (27) wird inhaltlich an einer für die Kritische Gesellschaftstheorie zentralen Methode ausgerichtet, der Ideologiekritik, die alle jene Bedingungen freilegen soll, die die Grundlage von Herrschaftsverhältnissen bilden. Die ideologiekritische Ausrichtung des Projektunterrichts soll also dessen emanzipative Wirkung sicherstellen.

Es ist nun kein großer Schritt, nicht nur die im Unterricht thematisierte Wirklichkeit ideologiekritisch zu durchleuchten, sondern den Unterschied selbst dieser Analyse zu unterwerfen. Mit diesem Schritt wird der Anspruch auf eine durchgängig herrschaftsfreie pädagogische Praxis gestellt und einzulösen versucht. Das ist die Geburtsstunde der antiautoritären Erziehung.

Die praktische Bedeutung der «kritisch-emanzipativen Wende» wird allgemein überschätzt. Mit ihr lässt sich zwar institutionelles Handeln ideologie- 
kritisch hinterfragen; unmittelbar unterrichtspraktisches Handeln, auf das es im Lehreralltag ja ankommt, allerdings nur bedingt begründen. Neben zeitgeschichtlichen Gründen hat die mangelnde unmittelbare unterrichtspraktische Relevanz einer kritischen Pädagogik also auch wissenschaftsimmanente Ursachen.

Auf konzeptioneller Ebene entfaltet die kritische Wende eine gewisse integrative Wirkung, indem sie Vorstellungen von fächerübergreifendem Unterricht und Projektunterricht zumindest publizistisch Geltung verschafft. Diese seit der Reformpädagogik zu Beginn des 20. Jahrhunderts in der pädagogischen Diskussion existenten Vorstellungen enthalten in sich natürlich auch Vorstellungen von erziehendem Unterricht, die einen weitgehenden disziplinenübergreifenden, also integrativen Anspruch beinhalten und auch in der fachdidaktischen Diskussion durchschlagen:

«So begann sich Ende der 60er Jahre das Lager der Geographen zu teilen. Ein traditioneller Stamm von Lehrern und Wissenschaftlern wollte die Eigenständigkeit des Schulfachs wieder hergestellt wissen .. Ein progressiverer Teil akzeptierte die Eingliederung der Geographie und wollte geographischen Unterricht in Form von Projekten durchgeführt sehen, in denen möglichst nahe an der Lebensrealität (,,alles ist komplex”) und nur so eng wie notwendig an der Fachwissenschaft (,,als blossem Teilbereich der Realität") gearbeitet werden sollte.» (28)

In der Unterrichtspraxis sind wenige methodische und didaktische Elemente einer emanzipativen Pädagogik und Didaktik aufgenommen worden. (29)

Praktisch wesentlich bedeutungsvoller als die Kritische Erziehungswissenschaft ist eine relativ schnell eintretende Entwicklung in der Erziehungswissenschaft resp. der Unterrichtspraxis, die hier als «psychologische Wende» bezeichnet werden soll. Mit dieser wird die Dialektik von materialer und formaler Bildung auf die Seite der formalen Qualifikationen hin aufgelöst. Hier entstehen neue Auffassungen des schulischen Lernens und der Sozialisation Jugendlicher, die das schulische Geschehen lediglich unter einer individualistischen Perspektive fassen, obwohl sie vordergründig popularisierte Argumentationsfetzen der kritischen Wende mitverwenden und sich einen progressiven Anschein geben. (30) Unter einer didaktischen Perspektive spielt der Bildungs- bzw. Lerninhalt bei solchen Ansätzen kaum noch eine Rolle. Zumeist wird die planmäBige, d.i. unterrichtliche Einführung in einen kulturellen Wissensbestand, als im Gegensatz zum Prozeß der Individuation stehend, gesehen. Unterricht wird in einen Prozeß der individuellen Autonomie aufgelöst, in dem ein qualifiziertes Verhältnis zur Welt nicht mehr zentral mitgedacht wird. Didaktik erscheint hier nur noch als eine Disziplin, in der dar- auf reflektiert wird, wie sich Unterrichtsinteraktionen auf die Autonomie von Individuen auswirken. Jüngst hat Dietrich Benner die letztere Entwicklung in den Zusammenhang der postmodernen Kritik an der Pädagogik gestellt. (31) Auch diese «Wende» tritt ein, ohne daß noch eine intensive Auseinandersetzung mit den Programmen der realistischen und der kritisch-emanzipativen Wende stattgefunden hätte.

Die didaktischen Auswirkungen dieser Auffassung von Unterricht sind in ihrer Ausschließlichkeit fatal, wenn auch nicht neu. Bereits die Reformpädagogik der 20er Jahre hatte ihre Bemühungen auf das «Wie des Unterrichtens» konzentriert. In der psychologistischen Wende wird der Unterricht nur noch als mit den in ihm stattfindenden Interaktionen bildungswirksam begriffen. (32) Damit aber wird zunächst die Methodik zum zentralen Gegenstand der Didaktik. Zugleich wird ihr Zusammenhang zu den zu erlernenden Inhalten nicht mehr systematisch aufgenommen und Methode auf Interaktionen reduziert.

\section{Atomisierte Unübersichtlichkeit}

Die hier nur erinnerte Entwicklung der Unterrichtswissenschaften in den letzten Dezennien hat zu einer eigentümlichen Unübersichtlichkeit (33) geführt. Wir stehen heute vor der Gefahr, daß das, was bisher als Einheitliches gedacht, konzipiert und in der Regel auch realisiert wurde, nämlich Unterricht, in atomisierte Teile auseinanderzubrechen droht. Nun existieren Unterrichtsdisziplinen gleichzeitig auf unterschiedlichen wissenschaftlichen Diskursen, dem der Geisteswissenschaft, dem des Positivismus, dem der Kritischen Theorie und dem des Postmodernismus atomisiert nebeneinander. (34) Es hat sich inter-und innerdisziplinär eine «neue Unübersichtlichkeit», wie Jürgen Habermas dies ausdrückt, herausgebildet.

Das wiederum dürfte zu einem verstärkten Auseinandertriften von «praktischen Disziplinen», etwa den Fachdidaktiken und den «allgemeinen Disziplinen», wie der allgemeinen Pädagogik oder der allgemeinen Didaktik und einer verstärkten Orientierung der Fachdidaktik an der Fachwissenschaft führen.

Treffen diese prognostizistischen Überlegungen zu, so dürften das Theorie-Praxis-Verhältnis und die Integration der Unterrichtswissenschaften problematischer werden. So ist zu erwarten, daß die allgemeindidaktische Diskussion noch von erziehungswissenschaftlichen Entwicklungen beeinflußt und vice versa wird, während die Fachdidaktik aufgrund ihres pragmatischeren Charakters sich vornehmlich an der Fachwissenschaft orientieren dürfte. Dies 
wiederum würde dann eine Auseinanderentwicklung von fachdidaktischer und allgemeindidaktischer Diskussion implizieren.

Dabei war es eines der zentralen Ziele der Bildungsreform, mit der Anbindung fachdidaktischer Forschung an wissenschaftliche (Ausbildungs-)Institutionen diese aus ihrer Einbindung in Handwerkelei und Ekklektizismus zu befreien und in der Anbindung an allgemeindidaktische und erziehungswissenschaftliche Forschung pädagogisch anspruchsvolle fachdidaktische Konzeptionen zu ermöglichen, die dann auch das entsprechende Material zur Lehrerausbildung hätten abgeben sollen. In dem $\mathrm{Maße}$, in dem die erziehungswissenschaftliche und die allgemeindidaktische Theorie selbst uneinheitlich werden, kann sich die Fachdidaktik nicht mehr an diesen orientieren.

Die zunehmende inner- und interdisziplinäre Desintegration der Unterrichtswissenschaften (35) hat dann unmittelbare Folgen für das Bildungssystem. Bildung zielt in ihrem Kern nämlich gerade auf die Synthese unterschiedlicher Diskurse bzw. Sprachspiele, also auf die Integration wissenschaftlicher Methoden, Vorgehens- und Denkweisen in den subjektiven Horizont von Menschen. (36) Diese zu leistende Synthese soll dann verantwortliches Handeln begründen. Das ist das wesentliche Ziel von Humboldts Universitätsreform (37) und entsprechend in den schulischen Horizont transformiert, auch das der schulischen Bildung. Das kann allerdings nur gelingen, wenn die Unterrichtsdisziplinen auch eine solche synthetisierende Leistung noch zulassen. Nur dann sind Lehrer in der Lage, einen Unterricht zu etablieren, der an dem Anspruch festhält, jenen zentralen Bereich der gesellschaftlichen und kulturellen Reproduktion, den wir Erziehung nennen, darzustellen.

Die dem Lehrer abverlangte sinnstiftende und damit Handeln orientierende Synthese aber bleibt dann bloßer Wunsch, wenn die wissenschaftlichen Diskurse und Disziplinen sich nicht mehr aufeinander beziehen, also das, was vom Lehrer verlangt wird, selbst nicht mehr zu leisten imstande sind.

Lyotard hat in einer die Diskussion um die Postmoderne auslösenden Studie diesen Zustand diagnostiziert: «Niemand spricht alle Sprachen (gemeint sind wissenschaftliche Diskurse, bzw. niemand beherrscht alle Sprachspiele - HJF), sie haben keine universelle Metasprache, der Entwurf des System-Subjekts ist ein Mißerfolg (gemeint ist hier z. B. der Marxsche Entwurf - HJF), der der Emanzipation hat mit der Wissenschaft nichts zu schaffen (gemeint ist hier die Aufklärung - HJF), man ist im Positivismus dieser oder jener vereinzelten Erkenntnis verstrickt, die Gelehrten sind Wissenschaftler, die Aufgaben eingeschränkter Forschung sind parzelläre Aufgaben geworden, die keiner beherrscht.» (38)
Diese skizzierte wissenschaftliche Unübersichtlichkeit, die dem Lehrer als gleichgültiges, d.i. damit auch beliebiges Nebeneinander völlig differierender Realitätskonstitutionen gegenübertritt, läßt nur die Möglichkeit, zu alltäglichen Orientierungen, zu dem, was man immer schon vor jeder Qualifikation wußte, Zuflucht zu nehmen, wenn man sinnhafte Praxis begründen will. (39) Die alltäglichen, unhinterfragten Orientierungen bestimmen so, welche praktische Bedeutung die atomisierten Elemente der verschiedenen Unterrichtsdisziplinen annehmen.

Damit würde sich der Lehrerberuf aus dem Programm der Moderne endgültig aussondern, noch ehe es gelungen wäre, wie entsprechende Untersuchungen zeigen (40), professionelle Praxis zu etablieren, die sich rational legitimieren könnte.

\section{Anmerkungen}

1 Unter Unterrichtsdisziplinen resp. -wissenschaften sollen alle Wissenschaftsdisziplinen verstanden werden, die an der Erforschung und zugleich dem Zustandekommen des Unterrichts beteiligt sind: Erziehungswissenschaft, Allgemeine und Fachdidaktik, Fachwissenschaft.

2 zit. n. Combe, A. Krisen im Lehrerberuf, Bensheim 1979, S. 46

${ }^{3}$ Nohl 1978, S. 3 f.

${ }^{4}$ Nohl 1978, S. 134

5 Nohl 1978, S. 136

6 Nohl 1978, S. 141

7 Die dann zur Methodenfreiheit des Lehrers geführt hat.

8 Nohl 1978, S. $140 \mathrm{f}$

${ }^{9}$ Gemeint sind die Lehrpläne der Länder der Bundesrepublik Deutschland, die 1960 in Kraft waren, die also in den 50er Jahren erarbeitet worden waren.

10 Schmidt 1972, S. 21. Allerdings weist Schrettenbrunner z. B. für den Geographieunterricht zu Recht darauf hin, daß gerade in der Bundesrepublik die Entpolitisierung der Unterrichtswissenschaften eine politische Funktion hatte. (Schrettenbrunner 1981, S. 10)

11 Groothoff 1972, S. 15 f.

12 Dies geschieht übrigens nicht in allen europäischen Ländern.

${ }^{13}$ Es entstehen nun die ersten Lehrstühle für Bildungsökonomie.

${ }_{14}$ Ruegg 1975, S. 184

15 Baethge 1974, S. 31

16 Becker 1977, S. 37

17 sh. Lüdtke 1973, Döring 1980 (!), Nöth 1976

$18 \mathrm{sh}$. Brezinka 1971. Ihm kommt das Verdienst zu, das Selbstverständnis eines wesentlichen Teils der Erziehungswissenschaftler auf den Begriff gebracht zu haben.

19 Klafki 1963 , S. 79

20 Heimann 1965, S. 19 
${ }^{21}$ Gemeint sind hier die Fachwissenschaften.

22 Heimann 1965, S. 39

23 Schmidt 1972, S. 22

24 Schmidt 1972, S. 23

25 Mollenhauer 1972 , S. 50 f.

26 Habermas 1971, S. 10, zit. n. Mollenhauer 1972, S. 51 Sh. auch den Beitrag von Schmidt-Wulfen in diesem Heft

27 Moderatere Formen dieses Anspruchs sind auch im fächerübergreifenden Unterricht enthalten.

28 Schrettenbrunner 1981, S. 13. Zu den Veränderungen des Geographieunterrichts sh. detailliert Hard 1976

29 Selbstverständlich sind sie vorgängig ihres ideologiekritischen Gehaltes entkleidet worden

30 sh. dazu Müller 1984, S. 75 ff.

31 sh. Benner 1986

32 Für die Lehrerbildung sh. Stiefel 1988

33 Ich übernehme hier den von Jürgen Habermas in die sozialwissenschaftliche Diskussion eingebrachten Begriff, um anzudeuten, daß die Lage der Unterrichtswissenschaften ein Moment allgemeinerer Entwicklungen darstellt.

34 Zwischen einer neopositivistisch verkürzten Didaktik und einer postmodernen Erziehungswissenschaft gibt es keinen Dialog mehr.

35 Dies natürlich nicht als Folge rein innerwissenschaftlicher Entwicklungen. Die Begriffe Moderne und Postmoderne verweisen aber auf gesellschaftliche und epochale Entwicklungen, denen sich die innerwissenschaftlichen Atomisierungstendenzen verdanken.

36 Der Begriff der Sprachspiele wird hier in Anlehnung an Wittgenstein verwandt. Er bedeutet hier, daß jede der erwähnten wissenschaftstheoretischen Richtungen ihre eigenen Regeln der Hervorbringung und der Legitimation ihres Wissens haben. (sh. Wittgenstein, 1982, 23)

37 sh. Humboldt 1957, S. 126

38 Lyotard 1986, S. 120

39 Oder aber einem postmodernen Relativismus das Wort zu reden, der dann jede Orientierung als gleich gültig und damit Sinn selbst auflöst.

40 sh. z. B. Füglister 1985

\section{Literatur}

BAETHGE, M. et. al. (1974): Produktion und Qualifikation, Hannover.

BECKER, E., WAGNER, B. (1977): Ökonomie der Bildung, Frankfurt/New York.

BENNER, D. (1986): Pädagogisches Wissen und pädagogisches Ethos. In: Vierteliahreszeitschrift für wissenschaftliche Pädagogik, 4, 507-518.

BREZINKA, W. (1971): Von der Pädagogik zur Erziehungswissenschaft. Eine Einführung in die Metatheorie der Erziehung. Weinheim.

COMBE, A. (1979): Krisen im Lehrerberuf, Bensheim.

DÖRING, K.W. (1980): Lehrerverhalten, Weinheim.

FÜGLISTER, P., BORN, R., FLÜCKIGER, V., KUSTER, H. (1985): Alltagstheorien von Berufsschullehrern über ihr unterrichtliches Handeln. Wissenschaftlicher Schlußbericht, Nationales Forschungsprogramm: Bildung und das Wirken in Gesellschaft und Beruf, Bern.

GROOTHOFF, H.H. (1965): Funktion und Rolle des Erziehers, München.

HEIMANN, P., GUNTER, O, SCHULZ, W. (1965): Unterricht, Analyse und Planung, Hannover.

HUMBOLDT, W., VON (1957): Über die innere und äußere Organisation der höheren wissenschaftlichen Anstalten in Berlin (1810). In: Wilhelm von Humboldt, Frankfurt.
KLAFKI, W. (1963): Studien zur Bildungstheorie und Didaktik, Weinheim.

LÜDTKE, H., Hrsg. (1973): Erzieher ohne Status, Heidelberg. LYOTARD, J.F. (1986): Das postmoderne Wissen, Graz, Wien.

MOLLENHAUER, K. (1972): Theorien zum Erziehungsprozeß, München

MÜLLER, F. (1984): Erziehung durch Unterricht oder: Die regulierende Funktion der Gegensätze. In: Busch F.W., Spelling, K., Hrsg., Schulleben heute, Materialien der 8. ATEEKonferenz, Oldenburg, S. 67-79.

NÖTH, W. (1976): Zur Theorie beruflicher Sozialisation. Dargestellt am Beruf des Grund- und Hauptschullehrers, Kronberg.

NOHL, H. (1978): Die pädagogische Bewegung in Deutschland und ihre Theorie, Frankfurt.

RUEGG, W. (1975): Funkkolleg Soziologie, Frankfurt.

SCHMIDT, A. (1972): Der Erdkundeunterricht, Bad Heilbrunn.

SCHRETTENBRUNNER, H. (1981): Situationsbericht: Grundlagen für die Durchführung des Geographieunterrichts. In: Schrettenbrunner, H. u. a., Geographieunterricht 5-10, Methodische Anregungen für Planung, Durchführung und Auswertung, München, S. 9-26.

STIEFEL, R. (1988): Persönlichkeitsbildung als Grundanliegen in der Lehrerbildung, Bern.

WITTGENSTEIN, L. (1982): Tractatus logico-philosophicus. Logisch-philosophische Abhandlung, Frankfurt. 